www.jmscr.igmpublication.org

Impact Factor (SJIF): 6.379

Index Copernicus Value: 79.54

ISSN (e)-2347-176x ISSN (p) 2455-0450

crossrefDOI: https://dx.doi.org/10.18535/jmscr/v6i12.56

Journal Of Medical Science And Clinical Research

IGM Publication

An Official Publication of IGM Publication

\title{
Heart Rate Variability in post-menopausal women Co related with Estradiol assay
}

\author{
Author \\ Dr Deepa Manuel \\ Assistant Professor, Dept of Physiology Karuna Medical College Vilayodi Chittur Palakkad 678103 Kerala, \\ India \\ Corresponding Author \\ Dr Deepa Manuel
}

Phone number: 8825758534, Email: deepamanuel@gmail.com

\begin{abstract}
Heart rate variability $(H R V)$ is a measure of the cyclical variations of beat-to-beat $(R R)$ intervals that reflects cardiac autonomic function and sympathovagal balance. HF component indicates parasympathetic activity and LF component indicates sympathetic activity. It has been proposed that there is sympathetic dominance of cardiac function in postmenopausal women.

The aim of the study was to compare HRV and Estradiol levels in premenopausal and postmenopausal women

After getting clearance from Institutional Human Ethics Committee (IHEC) and Department of Clinical Research and Bioethics (DCRB) the subjects who fulfilled the criteria for study was taken for ECG taking, $H R V$ analysis, and Estradiol assay.

The statistical analysis was done using SPSS software version 21 by unpaired Students t test. Analysis was done between the study group and control group. Both HRV and Estradiol levels were compared which gave the exact relationship between HRV and lack of Estrogen in the postmenopausal women.

$P$ value $<0.5$ was considered statistically significant.

The present study shows that there is sympathetic over activity in postmenopausal women denoted by elevated LF, and all the other parameters like Mean RR. SDNN, RMSSD, NN50, PNN50 were reduced showing increased sympathetic tone and increased propensity to cardiovascular diseases.

This effect will be due to reduced levels of Estrogen in Postmenopausal women

Keywords: Menopause, HRV, Autonomic functions.
\end{abstract}

\section{Introduction}

Heart rate variability (HRV) is a measure of the cyclical variations of beat-to-beat (RR) intervals that reflects cardiac autonomic function and sympathovagal balance. ${ }^{1}$ The power spectrum of HRV has two components. They are High Frequency component (HF) and Low Frequency component (LF). HF component is indicative of parasympathetic activity and LF component is indicative of sympathetic activity. LF/HF ratio reflects sympathovagal balance.

A predominance of sympathetic tone in cardiac activity induces tachycardia and reduced beat-tobeat variations, whereas parasympathetic nerve activity reduces heart rate and increases HRV. In general, higher HRV is desirable, and lower HRV 
has been found to be a significant independent predictor of cardiac mortality and morbidity. ${ }^{1}$ No specific therapy is currently available to improve the prognosis for patients with abnormal HR variability. Reduced HR variability has been most commonly associated with a risk of arrhythmic death.

Menopause is the permanent cessation of menstruation and is declining ovarian function as a result of exhaustion of the pool of primary follicles and associated decreased Estrogen production. $^{2}$

The question of whether the consequences of this decline are to be regarded as 'natural,' or as giving rise to a pathological state of oestrogen deficiency, is a controversial one ${ }^{2}$.

Prior to menopause estrogen balances sympathetic system and parasympathetic tone. During and after menopause diminished estrogen can result in sympathetic over-stimulation contributing to symptoms such as heart palpitations.

Experimental studies done by Xia Jun Du, Rudolph $\mathrm{A}^{3}$ et al have provided evidence that the autonomic nervous activity is modulated by oestrogen. Such modulation at central and peripheral levels tends to suppress sympathetic but elevate parasympathetic tone to the cardiovascular system. Thus, available data support the view that cardiovascular protection by oestrogen may, at least in part, be mediated by its influence on autonomic nervous function.

In healthy young women, the cardiovascular system is regulated by high vagal cardiac tone and lower sympathetic cardiovascular control. With aging in healthy women, sympathetic control predominates. $^{4}$

Although the rise in cardiovascular risk factors after menopause is a known factor, the cause is debated .Studies done by Renata Cifcova ${ }^{5}$ et al states that the increased BMI after menopause is the cause for increased cardiovascular riskfactors. ${ }^{5}$ Studies done by Edoardo Casiglia ${ }^{6}$ says that increase in cardiovascular risk factors after menopause is due to ageing and not due to menopause. ${ }^{7}$
Most of the studies agree with the fact that increased cardiovascular risk factors are due to Estrogen lack factor which is associated with low $\mathrm{HF}$ and high LF .Much studies are not done in Indian population. Nirmala et $\mathrm{al}^{7}$ compared HRV in Reproductive and Postmenopausal women. But here age can be a cofounding factor ie. Low HRV can be due to ageing apart from Estrogen lack factor. In our study we tried to rectify the drawback by selecting premenopausal women above 45 years age.

In this study we also have done Estradiol assay and we have correlated Estradiol lack factor and low HRV.

\section{Materials and Methodology}

ECG recording and HRV analysis

After getting clearance from Institutional Human Ethics Committee (IHEC) and Department of Clinical Research and Bioethics (DCRB) the subjects who fulfilled the crireria for study was taken for ECG taking, HRV analysis, and Estradiol assay.

Twenty two premenopausal women and twenty three postmenopausal women were taken for study after taking informed consent. Postmenopausal women of age 55-70 with no vaginal bleeding ,not on HRT were included for study. Premenopausal women between 35-44 having normal menstrual cycles and not on oral contraceptive pills were taken for study.

Females who were diabetic, hypertensive who were on drugs affecting Autonomic nervous system and those who were having BMI less than 18.5 and more than 25 were excluded from both the groups.

\section{Body Mass Index}

All the participants were measured for height and weight. From this BMI was obtained by dividing weight in kilogram by square of the height in meters. BMI of 18.5 to 24.9 were graded as normal, 25 to 29.9 were graded as overweight and more than 30 as obese. Only the participants with normal BMI were included in the study. 


\section{ECG Recording}

Informed consent was taken from all the subjects before taking ECG.

The parameters recorded were Resting Heart Rate, Body mass index and ECG recording. Twenty two pre menopausal women and twenty three post menopausal women were taken for the study.

ECG was recorded for a period of 5 minutes. It was recorded by placing disposable adhesion electrodes on the pattern of lead II configuration. Negative electrode was connected to Right arm, positive electrode to left arm reference electrode on left foot and ground electrode on right foot. Baseline electrocardiogram were obtained from all subjects and those with abnormal baseline ECG were excluded.

ECG was recorded using the computerized physiograph NIVIQURE Digital ECG recorder in lead II for a period for 5 minutes. RR intervals were obtained after clearance of noise and baseline fluctuations by digital filters. Those who had ectopic beats were excluded from the study.

The Time domain parameters calculated are

- $\quad$ Mean RR (NN).

- SDNN standard deviation of all NN intervals.

- RMSSD the square root of the mean squared difference of successive NNs that differ by more than $50 \mathrm{~ms}$.

- $\mathrm{PNN}_{50}$ the proportions of NN50 divided by total number of NNs.

\section{Frequency domain parameters calculated are}

- Low frequency power(LF) -the LF spectrum is evaluated in the range from 0.04 to $0.15 \mathrm{~Hz}$ This band can reflect mainly sympathetic tone.

- High frequency power-The HF power spectrum is evaluated in the range from 0.15 to $0.4 \mathrm{~Hz}$. This band reflects parasympathetic tone.

- $\mathrm{LF} / \mathrm{HF}$ power ratio- The $\mathrm{LF} / \mathrm{HF}$ ratio is used to indicate balance between sympathetic and parasympathetic tone. A decrease in the score indicate either increase in parasympathetic or decrease in sympathetic tone.

HRV analysis was done by feeding this RR interval notepad file to HRV analysis software version 1.1 from Biomedical Signal Analysis group, Department of Applied Physics, University of Kuopio, Finland.

\section{Estradiol Assay}

After taking ECG, 3ml blood sample was taken for Estradiol assay. The bood sample was centrifuged $3000 \mathrm{rpm}$ for 15 minutes, serum was separated, stored in the deep freezer and later Estradiol assay was done using Elecsys kit.

The Elecsys Estradiol 2 assay employs a competitive test principle using a polyclonal antibody specifically directed against $17 \quad \beta$ estradiol. Endogenous estradiol released from the sample by mesterolone competes with the added estradiol derivative labeled with a ruthenium complex for the binding sites on the biotinylated antibody.

\section{Statistical analysis}

The statistical analysis was done using SPSS software version 21, by unpaired Students t test. Analysis was done between the study group and control group. Both HRV and Estradiol levels were compared which gave the exact relationship between HRV and lack of Estrogen in the postmenopausal women.

\section{Results}

The HRV parameters (Time domain measures and frequency domain measures) and Esradiol values were compared between the cases (postmenopausal women) and the controls (perimenopausal women).

\section{Baseline characteristics of Postmenopausal and Perimenopausal age group}

Age

Age of the postmenopausal group was 50.50 \pm 5.33 and that of the controls were $39.70 \pm 2.70$.There 
was a significant increase in age of postmenopausal women with a $\mathrm{p}$ value of 0.0001 .

\section{BMI}

BMI of postmenopausal group was $24.55 \pm 2.24$ and that of controls were 23.65 \pm 2.01 . There was no significant relationship between BMI of cases and controls with a $\mathrm{p}$ value of 0.1883 .

\section{Heart Rate}

The Heart rate of postmenopausal group was $84.05 \pm 10.66$ and that of the perimenopausal group was $71.70 \pm 9.48$. There was a significant increase in Heart Rate of postmenopausal women with a $p$ value of 0.0004 .

\section{Comparison of frequency domain measures} of the postmenopausal and perimenopausal group.

\section{Low power frequency (LF) in ms}

LF of postmenopausal women was $29.05 \pm 16.25$ and that of the perimenopausal women was $66.50 \pm 7.177$. There was significant difference between LF powers with a $p$ value of 0.0028. The values were increased for the controls.

\section{High power frequency in $\mathrm{ms}$}

HF of the postmenopausal was $345.75 \pm 128.14$ and that of perimenopausal women was 511.65 \pm 233.86 . There was significant decrease in postmenopausal women for HF powers with a $\mathrm{p}$ value of 0.0084 .

\section{LF/HF ratio}

$\mathrm{LF} / \mathrm{HF}$ of postmenopausal was $1.063 \pm 0.505$ and that of perimenopausal women was $0.431 \pm 0.145$. There was significant increase for LF/HF ratios in the postmenopausal age group with a $\mathrm{p}$ value less than 0.0001 .

\section{Low power frequency in normalized units (LF nu)}

LF nu of the postmenopausal women was $40.220 \pm 7.177$ and the perimenopausal women was $31.040 \pm 10.650$. There was significant increase in postmenopausal women for $\mathrm{LF}$ nu with a $\mathrm{p}$ value of 0.0028 .
High power frequency in normalized units (HF nu)

HF nu of the postmenopausal women was $41.735 \pm$ 10.64 and the perimenopausal women was $61.0550 \pm 13.32$. There was significant decrease in postmenopausal women for $\mathrm{HF}$ nu with a $\mathrm{p}$ value of 0.0001 .

\section{Comparison of time domain measures of postmenopausal and perimenopausal group. Mean RR}

Mean RR of the postmenopausal group was $0.59645 \pm 0.196$ and the perimenopausal group was $0.78995 \pm 0.4274$. There was significant decrease in postmenopausal women for Mean RR with a $p$ value of 0.001 .

\section{SDNN}

SDNN of the postmenopausal was $0.05670 \pm 0.625$ and that of the controls was $0.24930 \pm 0.27578$. There was significant decrease in postmenopausal women for SDNN with a $\mathrm{p}$ value of 0.0010 .

\section{RMSSD}

RMSSD of the postmenopausal women was $63.450 \pm 36.220$ and that of the perimenopausal group was 92.220 \pm 43.698 . There was a significant decrease in postmenopausl women for RMSSD with a $\mathrm{p}$ value of 0.0292 .

NN50

NN50 of postmenopausal women was $21.4 \pm 11.5230$ and that of the controls was $38.69 \pm 12.7$. There was a significant decrease in postmenopausal women for NN50 with a $\mathrm{p}$ value of 0.0001 .

$\mathbf{p N N}_{\mathbf{5 0}}$

$\mathrm{pNN}_{50}$ of the postmenopausal women was $10.930 \pm 6.418$ and that of the controls was 28.970 \pm 9.729 . There was a significant decrease in postmenopausal women for $\mathrm{pNN}_{50}$ with a $\mathrm{p}$ value less than 0.0001 . 
Table 1 Comparison of Baseline characteristics of Postmenopausal and premenopausal group

\begin{tabular}{|l|c|c|c|}
\hline Parameter & Group & Mean \pm SD & P Value \\
\hline \multirow{3}{*}{ Age } & Cases & $50.50 \pm 5.33$ & \multirow{2}{*}{$0.0001^{* * * *}$} \\
\cline { 2 - 3 } & Controls & $39.70 \pm 2.70$ & \\
\hline \multirow{3}{*}{ BMI } & Cases & $24.55 \pm 2.24$ & \multirow{2}{*}{$0.1883^{\mathrm{NS}}$} \\
\cline { 2 - 3 } Heart rate & Controls & $23.65 \pm 2.01$ & \\
\cline { 2 - 3 } 0 & Cases & $84.05 \pm 10.66$ & \\
\cline { 2 - 3 } & Controls & $71.70 \pm 9.48$ & \\
\hline
\end{tabular}

NS Not significant ${ }^{*}$ Significant

${ }^{* *}$ Very significant ${ }^{* * *}$ Extremly significant

Table 2 Comparison of Frequency domain measures of postmenopausal and premenopausal women

\begin{tabular}{|l|c|c|c|}
\hline Parameter & Group & Mean \pm SD & \multirow{2}{*}{ P value } \\
\hline \multirow{3}{*}{ LF nu } & Cases & $40.220 \pm 7.170$ & \multirow{2}{*}{$0.0028^{* *}$} \\
\cline { 2 - 3 } & Controls & $31.640 \pm 10-65$ & \multirow{2}{*}{$<0.0001^{* * *}$} \\
\hline \multirow{3}{*}{ HF nu } & Cases & $41.7315 \pm 10.64$ & \multirow{2}{*}{$<0.0001^{* * *}$} \\
\cline { 2 - 3 } LF/HF ratio & Controls & $61.0550 \pm 13.32$ & \multirow{2}{*}{$0.0004^{* * *}$} \\
\hline \multirow{3}{*}{ LF in ms } & Cases & $1.063 \pm 0.505$ & \multirow{2}{*}{$0.0084^{* *}$} \\
\hline \multirow{3}{*}{ HF in ms } & Controls & $0.431 \pm 0.145$ & \\
\cline { 2 - 3 } & Cases & $29.05 \pm 16.25$ & \\
\cline { 2 - 3 } & Controls & $66.50 \pm 40.28$ & \multicolumn{2}{|c}{} \\
\cline { 2 - 3 } & Cases & $345.75 \pm 128.14$ & \multicolumn{2}{|c}{} \\
\hline
\end{tabular}

NS Not significant * Significant

Very significant $\quad{ }^{* * *}$ Extremly significant

Table 3 Comparison of time domain measures of postmenopausal and premenopausal group

\begin{tabular}{|c|c|c|c|}
\hline Parameter & Group & Mean \pm SD & $\mathrm{P}$ value \\
\hline \multirow[b]{2}{*}{ Mean RR } & Cases & $0.5964 \pm 0.196$ & \multirow[b]{2}{*}{$0.0010^{* *}$} \\
\hline & Controls & $0.78995 \pm 0.4274$ & \\
\hline \multirow[b]{2}{*}{ SDNN } & Cases & $0.5670 \pm 0.625$ & \multirow[b]{2}{*}{$0.0033^{* *}$} \\
\hline & Controls & $0.24980 \pm 0.27378$ & \\
\hline \multirow[b]{2}{*}{ RMSSD } & Cases & $63.450 \pm 36.220$ & \multirow[b]{2}{*}{$0.0292^{*}$} \\
\hline & Controls & $92.220 \pm 43.698$ & \\
\hline \multirow[b]{2}{*}{ NN50 } & Cases & $21.4 \pm 11.5230$ & \multirow[b]{2}{*}{$<0.0001^{* * *}$} \\
\hline & Controls & $38.69 \pm 12.7$ & \\
\hline \multirow[b]{2}{*}{ pNN50 } & Cases & $10.930 \pm 6.418$ & \multirow[b]{2}{*}{$<0.0001^{* * *}$} \\
\hline & Controls & $28.970 \pm 12.7$ & \\
\hline
\end{tabular}

NS Not significant ${ }^{*}$ Significant

${ }^{* * *}$ Very significant ${ }^{* * * *}$ Extremly significant

Table 4 Comparison of Estradiol levels of postmenopausal and perimenopausl group in pg/ml.

\begin{tabular}{|l|c|c|c|}
\hline Parameter & Group & Mean \pm SD & P value \\
\hline \multirow{3}{*}{ Estradiol } & Cases & $10.96 \pm 6.41$ & \multirow{2}{*}{$0.0001^{* * *}$} \\
\cline { 2 - 3 } & Controls & $86.65 \pm 11.18$ & \multirow{2}{*}{ Con } \\
\hline
\end{tabular}

NS Not significant ${ }^{*}$ Significant

${ }^{* *}$ Very significant $\quad{ }^{* * *}$ Extremly significant

\section{Discussion}

Menopause is permanent cessation of menstruation as a result of the irreversible loss of a number of ovarian functions including ovulation and oestrogen production. The ovarian functions gradually become diminished and so, the oestrogen production from the granulosa cells of the ovary reduces,

It has long been suggested that oestrogen protects against atherosclerosis, because the incidence of 
cardiovascular disease is lower in women than in men in the reproductive age group. In menopausal women, the risk of cardiovascular diseases gradually increases due to the lack of the oestrogenic protective effects and the incidence of cardiovascular disease is equal to that in men. It is not so in premenopausal women because of the highly circulating oestradiol levels.

Menopause is a transition phase from reproductive life to nonreproductive life in an adult lady. The estrogen level reduces in postmenopausal period compared to premenopausal period. The lack of estrogen is said to be the cause of menopausal symptoms like hot flushes.

The postmenopausal period is also charecterised by increased cardiovascular risk factors like hypercholesterolemia, obesity, atherosclerosis. Researches shows that parasympathetic tone decreases in postmenopausal women and sympathetic tone dominates.

Heart Rate Variability is a research tool to asess sympathovagal balance. The increased HRV parameters is found to be desirable and low HRV parameters are found to be predictor of mortality and morbidity.

Autonomic modulation of $\mathrm{HR}$ has been documented as progressively declining with aging. This process has been demonstrated both by the reduction of vagal activity indices and of indices that reflect sympathetic activity on the heart . Since significantly decreased temporal indices of HRV were observed in postmenopausal women our results agree with the literature. ${ }^{8}$

From other studies it is documented that parasympathetic function decreases after menopause.

It was established by Latifa Afrin Dill Naher ${ }^{9}$ et al that parasympathetic function was lower in postmenopausal women which may be related to decreased Estrogen levels.

Shaher $\mathrm{Levi}^{4}$ et al also investigated the effect of healthy ageing on cardiovascular homeostatic mechanisms. They also found out that parasympathetic control dominates in young women and sympathetic tone dominates in postmenopausal women.
Our study also got the same result that parasympathetic tone decreases in postmenopausal women, which was shown by low levels of HF compared to the controls the premenopausal women.

The present study shows that there is sympathetic over activity in postmenopausal women denoted by elevated LF, and all the other parameters like Mean RR. SDNN, RMSSD, NN50, PNN50 were reduced showing increased sympathetic tone and increased propensity to cardiovascular diseases.

Our study also shows a decreased levels of Estradiol in postmenopausal women which could be the reason for reduced parasympathetic tone .

Although there is increased risk of cardiovascular diseases certain studies shows that the HRV parameters can be improved after a certain period of exercise treatment. K. P. Davy, N. L. Miniclier, J. A. Taylor ${ }^{10}$ et al. tested the hypothesis that HRV at rest is greater in physically active compared with less active postmenopausal women. The results of the investigation indicate that physically active postmenopausal women demonstrate higher levels of HRV compared with age-matched, less active women.

On the basis of the study Estrogen appears to be the causative factors for reduced Heart Rate Variability, we don't recommend HRT although HRT is proved to increase Heart Rate Variability parameters. The reason is that it can cause Endometrial carcinoma.

We discussed the harmful effects of Hormone Replacement Treatment, but phytoestrogens can be used as an alternative for postmenopausal women suffering from hot flushes as well as cardiovascular diseases. The studies on this phytoestrogens does not show a valid proof so we recommend that researches should be taken on this aspect-whether phytoestrogens are an alternative for Hormone replacement treatment.

There is evidence based on other studies that vegetarian diet is found to be associated with increased HRV levels compared to non vegetarians. $^{32}$ 


\section{Limitations of the study}

Although we tried to avoid the confounding factor age, it seems to interfere with the results because ageing can itself cause decreased HRV parameters.

We tried to take women of age around 45 for the control group but there is significant difference between cases and controls with a $p$ value of 0.0001 .

We measured Estradiol levels but did not take into account other Estrogens which are predominant in postmenopausal women.

Small sample size is a limiting factor for this study.

\section{Conclusion}

Our study shows that there is reduced parasympathetic tone and increased sympathetic tone in postmenopausal women. The cause is found to be the reduced levels of Estradiol levels as well as the increased age of the postmenopausal women.

It is shown that healthy lifestyles, adequate amount of nutrients like Calcium and Vit D, maintaining mental health, adequate exercise, predominantly vegetarian diet is found to be of benefit for postmenopausal ladies to lead a healthy life.

We don't recommend Hormone Replacement Therapy solely for the purpose of improving cardiovascular outcome because of other risk factors. Researches on Phytoestrogens should be taken up for improving morbidity as well as mortality in postmenopausal women.

Source of support- PG FUND for postgraduates from PSG IMS\&R

\section{References}

1. E Ammandola, V RussoL Politano L Sant Angelo "Is Heart Rate variability a valid parameter to predict sudden death in patients in Becker's Muscular Dystrophy? Heart2006 :92,1686-1687
2. Endocrinology of the menopause. Won-whe Kim Department of Obstetrics and Gynaecology, School of Medicine, Pusan National University, Pusan, Republic of korea

3. Xia Jun Du, Rudolph A.Anthony $\mathrm{M}$ et al Cardiovascular protection by Estrogen is partly mediated through modulation of ANS Oxford Journals Vol 30. Issue 2 p 161-165

4. Shaher Lavi Ori Navo, Israel Thaler Rimma Rosenfeld et al " Effect of Aging on Cardiovascular System in healthy women" AJP Vol 292 no,2 P R788- R 793

5. .Cifkova, Renata; Pitha, Jan; Lejskova, Magdalena; Lanska, Vera; Zecova, Silvia et al, Journal of Hypertension "Menopause does not cause increase in Blood Pressure" 2 nd October 2008. vol 26 (10) 1976-1982

6. Casiglia, Edoardo; Tikhonoff, Valérie; Caffi, Sandro; Bascelli et alJournal of Hypertension vol26 (10 ) p 1983-1992 Eng J Med 1999340 :1801-1811

7. Nirmala Natarajan, Lakshmipriya Paneerselvam, Latha Radhakrishnan et al Int J med Sci Public Health "Heart Rate Variability among Reproductive and Postmenopausal women.” 2015;4(8):1132-1135

8. VF.C Neves, M F Silva "Autonomic modulation of Heart Rate of young and postmenopausal women undergoing Estrogen therapy. Braz J Med Biol Res April 2007 , vol 40 (4) 491-499

9. Latifa Afrin Dill Naher, Noorzahan Begum, Shelina Begum, Sultana Ferdousi et al. Parasympathetic function status in postmenopausal women. Journal of Bangladesh Society of Physiologist. Vol 4 no.1(2009)

10. K P Davy, N L Miniclier, J A Taylor Elevated HRV in physically active postmenopausal women: a cardiopro-tective effect? AJP Heart and Circulatiry physiology Vol 271 Issue $2 \mathrm{p}$ 455-46. 